\title{
Possible Implication of Intermolecular Epitope Spreading in the Production of Anti-Glomerular Basement Membrane Antibody in Anti-Neutrophil Cytoplasmic Antibody-associated Vasculitis
}

\section{Yuka Nishibata}

Hokkaido University: Hokkaido Daigaku

\section{Mayu Nonokawa}

Hokkaido University: Hokkaido Daigaku

\section{Yuto Tamura}

Hokkaido University: Hokkaido Daigaku

Rio Higashi

Hokkaido University: Hokkaido Daigaku

Ku Suzuki

Hokkaido University: Hokkaido Daigaku

Hideyuki Hayashi

Hokkaido University: Hokkaido Daigaku

\section{Sakiko Masuda}

Hokkaido University: Hokkaido Daigaku

\section{Daigo Nakazawa}

Hokkaido University: Hokkaido Daigaku

\section{Satoshi Tanaka}

Hokkaido University: Hokkaido Daigaku

\section{Utano Tomaru}

Hokkaido University: Hokkaido Daigaku

Akihiro Ishizu ( $\square$ aishizu@med.hokudai.ac.jp )

Hokkaido University https://orcid.org/0000-0002-7084-9734

\section{Research article}

Keywords: Anti-neutrophil cytoplasmic antibody (ANCA), ANCA-associated vasculitis, Anti-glomerular basement membrane (GBM) antibody, Intermolecular epitope spreading

Posted Date: March 18th, 2021 
DOl: https://doi.org/10.21203/rs.3.rs-322082/v1

License: (c) (1) This work is licensed under a Creative Commons Attribution 4.0 International License. Read Full License

Version of Record: A version of this preprint was published at Clinical and Experimental Rheumatology on June 30th, 2021. See the published version at https://doi.org/10.55563/clinexprheumatol/6oq9du. 


\section{Abstract}

Objective

Anti-neutrophil cytoplasmic antibody (ANCA)-associated vasculitis (AAV) is sometimes complicated by anti-glomerular basement membrane (GBM) disease. Proteases, including elastase, released from neutrophils activated by ANCA are implicated in the pathogenesis of AAV. Epitopes of anti-GBM antibody exist in the a3-subunit non-collagenous (NC1) domain of collagen type IV [Col (IV)]. This region, called a3(IV)NC1, is structurally cryptic. This study aimed to determine the production mechanism of anti-GBM antibody in AAV.

Methods

We first examined whether a3(IV)NC1 could be revealed by the digestion of formalin-fixed, paraffinembedded (FFPE) normal kidney sections and Col (IV) by proteases, including neutrophil elastase, using immunohistochemistry (IHC) and enzyme-linked immunosorbent assay (ELISA). Next, the reveal of a3(IV)NC1 and the infiltration of $\mathrm{CD} 11 \mathrm{c}^{+}$macrophages in the affected kidneys were evaluated by $\mathrm{IHC}$ and immunofluorescent staining using FFPE sections. Finally, the production of anti-GBM antibody in AAV rats was determined by ELISA.

Results

a3(IV)NC1 was revealed by the digestion of FFPE normal kidney sections and Col (IV) by proteases. Although the reveal of a3(IV)NC1 was observed in sclerotic glomeruli regardless of causative diseases,

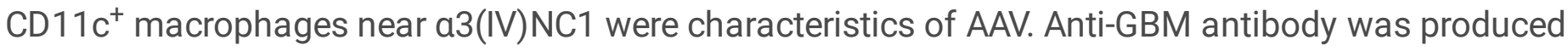
subsequent to ANCA in some AAV rats. IHC demonstrated the reveal of a3(IV)NC1 in affected renal tissues and the infiltration of $\mathrm{CD} 11 \mathrm{c}^{+}$macrophages around the sites.

Conclusion

The collective findings suggest that, in AAV, proteases released from neutrophils activated by ANCA digest Col (IV) and result in the reveal of a3(IV)NC1, CD11 $\mathrm{c}^{+}$macrophages present GBM epitopes, and then the host's immune system produce anti-GBM antibody.

\section{Introduction}

Anti-neutrophil cytoplasmic antibody (ANCA)-associated vasculitis (AAV) is a systemic small vessel vasculitis accompanied by the presence of ANCA in the serum [1]. This disease entity includes microscopic polyangiitis (MPA), granulomatosis with polyangiitis, and eosinophilic granulomatosis with polyangiitis. The major antigens of ANCA are myeloperoxidase (MPO) and proteinase 3. The ANCAmediated activation of neutrophils is critically implicated in the pathogenesis of AAV [2], and the neutrophils activated by ANCA can release proteases, including elastase. 
Anti-glomerular basement membrane (GBM) disease is an organ-specific autoimmune disorder characterized by diffuse necrotizing crescentic glomerulonephritis and pulmonary hemorrhage with the production of anti-GBM antibody [3]. This autoantibody binds to collagen type IV [Col (IV)] that constitutes the glomerular and alveolar basement membranes. Col (IV) is composed of three a-subunits, and it can be divided into three domains, namely, 7s domain, collagenous domain, and non-collagenous (NC1) domain [4]. The collagenous domain is a triple-helix bundle of a-subunits. There are two major combinations of a-subunits, namely, a1, a1, and a2 (1-1-2) and a3, a4, and a5 (3-4-5). Col (IV), which contains the 1-1-2 a-subunits, is distributed widely in systemic organs, whereas Col (IV), which contains the 3-4-5 a-subunits, exists in limited organs, including the kidneys and lungs.

The major epitopes recognized by anti-GBM antibody, namely EA and EB, are present in the a3-subunit NC1 domain [5]. a3(IV)NC1 [a3-subunit NC1 domain of Col (IV)], which covers these epitopes, is structurally cryptic, owing to the hexamer formation-sulphilimine linkage of 3-4-5 a-subunit trimers-of the NC1 domain, and is revealed by cleaving the sulphilimine linkage [6]. In cases where the hexamer formation of Col (IV) NC1 domain is dissociated, anti-GBM antibody can bind to the epitopes and then the complement cascade is activated, resulting in complement-dependent tissue destruction.

Approximately $30 \%$ of patients with anti-GBM disease possess ANCA in the serum and $5 \%$ of AAV patients are positive for circulating anti-GBM antibody [7]. Olson et al. have noted that, in some patients with anti-GBM disease, ANCA has been produced at least 1 year before the detection of anti-GBM antibody [8]. These findings suggest the association between AAV and anti-GBM disease.

It has been shown by immunofluorescent (IF) staining that sera of patients with anti-GBM disease can bind to GBM in frozen sections of normal kidneys in a restrictive pattern [9]. In contrast, in immunohistochemistry (IHC) using formalin-fixed, paraffin-embedded (FFPE) normal kidney sections, anti-a3(IV)NC1 monoclonal antibody (mAb) did not bind to the epitope without an appropriate antigen retrieval [10]. This controversy can be explained by the difference in fixation-frozen or formalin fixationand/or in the epitopes recognized by the patient's sera and the mAb. Naito et al. have demonstrated that heating under acidic condition $(0.2 \mathrm{M} \mathrm{HCl}, \mathrm{pH} 0.9)$ is required to retrieve the a3(IV)NC1 epitope from FFPE normal kidney sections [10]. It is considered that protein refolding during the cooling process after heating is inhibited under acidic condition, resulting in the reveal of the cryptic epitope [11]. Interestingly, protease treatment could also retrieve a3(IV)NC1 from FFPE normal kidney sections [12].

Based on these evidence, it was hypothesized that proteases released from neutrophils activated by ANCA will cut peptide bonds next to the sulphilimine linkage between 3-4-5 a-subunit trimers of the Col (IV) NC1 domain, which will similarly lead to exposure of the cryptic epitopes, and that consequently, the host's immune system will recognize the revealed GBM epitopes.

\section{Materials And Methods}

\section{Human materials}


FFPE kidney tissues of patients with or without renal disease, which were obtained by autopsy, were used. Renal diseases include MPA ( $n=4)$, IgA nephropathy (IgAN; $n=2)$, and hypertensive arteriosclerosis $(n=3)$. Kidney tissues of a patient who died of Ewing's sarcoma but did not manifest renal involvement were used as normal kidney tissues, as there was no pathological finding. All patients were negative for antiGBM antibody. Peripheral blood $(10 \mathrm{ml})$ was obtained from a healthy volunteer after acquisition of written informed consent.

\section{Rats}

Wistar-Kyoto (WKY) rats (3 weeks old, male) were purchased from Sankyo Laboratory (Sapporo, Japan) and maintained under specific pathogen-free condition.

\section{Reagents}

Col (IV) derived from human placenta that includes a3(IV)NC1, proteases derived from Streptomyces griseus (S. griseus), pertussis toxin, and phorbol myristate acetate (PMA) were purchased from SigmaAldrich (St. Louis, MO, USA). Neutrophil elastase (NE) was purchased from Abcam (Cambridge, UK). Recombinant human a3(IV)NC1 and MPO light chain were purchased from Cloud-Clone (Katy, TX, USA). Human native MPO was purchased from RayBiotech (Peachtree Corners, GA, USA) and Elastin Products Company (Owensville, MO, USA).

\section{Antibodies}

Antibodies used as anti-human primary antibodies include rat anti-a3(IV)NC1 mAb (clone H31, IgG2a; Chondrex, Redmond, WA, USA), rabbit anti-a3(IV)NC1 polyclonal antibody (pAb; Cloud-Clone), mouse antitumstatin, a3(IV)NC1 mAb (clone MAB7546, IgG1; Bio-Techne, Minneapolis, MN, USA), rabbit anti-CD11c pAb (Thermo Fisher Scientific, Rockford, IL, USA), and mouse anti-CD68 mAb (clone KP1, IgG1; Abcam). Antibodies used as secondary antibodies include horseradish peroxidase (HRP)-conjugated goat anti-rat IgG1 (Bethyl Laboratories, Montgomery, TX, USA), IgG2a (Bio-Rad Laboratories, Hercules, CA, USA), and IgG2b (Bio-Rad Laboratories) antibodies, HRP-conjugated goat anti-rat IgG antibody (Bethyl Laboratories), HRP-conjugated goat anti-rabbit IgG antibody (Jackson ImmunoResearch, West Grove, PA, USA), HRP-conjugated goat anti-mouse IgG antibody (SeraCare Life Sciences, Milford, MA, USA), fluorescein isothiocyanate (FITC)-conjugated rabbit anti-rat IgG antibody (Dako, Glostrup, Denmark), Alexa Fluor 594-conjugated goat anti-rabbit IgG antibody (Abcam), and Alexa Fluor 488-conjugated goat anti-mouse IgG antibody (Abcam).

\section{Antigen retrieval from FFPE normal kidney sections}

FFPE normal kidney sections were cut into $4 \mu \mathrm{m}$ sections, mounted on silane-coated glass slides, deparaffinized with xylene, and then autoclaved at $121^{\circ} \mathrm{C}$ under acidic condition $(0.2 \mathrm{M} \mathrm{HCl}, \mathrm{pH} 0.9,6$ min), neutral condition (citrate buffer, pH 6.0, 10 min), or alkaline condition (Tris-EDTA, pH 9.0, 20 min). Sections that did not undergo heating were used as controls. In other experiments, FFPE normal kidney sections were deparaffinized with xylene, heated under neutral condition, and then exposed to proteases 
derived from S. griseus $(0.5 \mathrm{mg} / \mathrm{ml})$ for $30 \mathrm{~min}$ at $37^{\circ} \mathrm{C}$. After rinsing in running tap water for $5 \mathrm{~min}$, the sections were subjected to IHC for a3(IV)NC1.

\section{IHC for a3(IV)NC1 using FFPE normal kidney sections}

After antigen retrieval, the sections were exposed to $3 \%$ hydrogen peroxide $\left(\mathrm{H}_{2} \mathrm{O}_{2}\right)$-methanol to inactivate endogenous peroxidase in the tissues followed by blocking the non-specific binding of antibodies and then allowed to react with rat anti-a3(IV)NC1 mAb (1:1000) for $1 \mathrm{~h}$ at room temperature (RT). After rinsing with phosphate-buffered saline (PBS), the sections were next made to react with the HRP-conjugated goat anti-rat IgG2a antibody (1:1000) for $1 \mathrm{~h}$ at RT. After rinsing with PBS, the HRP activity on tissue sections was detected by $3,3 \bar{c}$-diaminobenzidine (DAB) substrates followed by counterstaining with Mayer's hematoxylin dye.

\section{Digestion of Col (IV) by proteases}

Col (IV) $(1 \mathrm{mg} / \mathrm{ml})$ was mixed with an equal volume of proteases derived from S. griseus at final concentrations of $0,1.25,12.5,125$, and $1250 \mu \mathrm{g} / \mathrm{ml}$ or NE at final concentrations of $0,125,250,500$, 1000 , and $2000 \mu \mathrm{U} / \mathrm{ml}$ for $30 \mathrm{~min}$ at $37^{\circ} \mathrm{C}$. Thereafter, the solution was heated for $15 \mathrm{~min}$ at $80^{\circ} \mathrm{C}$ to inactivate the enzymes. Samples were subjected to enzyme-linked immunosorbent assay (ELISA) to detect a3(IV)NC1.

\section{Detection of a3(IV)NC1 by ELISA}

Wells of 96-well plates (Thermo Fisher Scientific) were coated with $5 \mu \mathrm{g} / \mathrm{ml}$ rat anti-a3(IV)NC1 mAb (50 $\mu \mathrm{l} /$ well) overnight at $4{ }^{\circ} \mathrm{C}$ and then washed four times with PBS containing $0.05 \%$ Tween 20 (PBS-T) and soaked in PBS-T containing $1 \%$ skim milk ( $150 \mu \mathrm{l} /$ well) for $1 \mathrm{~h}$ at RT to block the non-specific binding of antibodies. After the removal of the blocking solution from the wells, positive control [PC; $1.25 \mu \mathrm{g} / \mathrm{ml}$ recombinant human a3(IV)NC1] or sample solution ( $50 \mu \mathrm{l} /$ well) was added into the wells and then incubated for $1 \mathrm{~h}$ at RT. After washing with PBS-T, the wells were next incubated with $1 \mu \mathrm{g} / \mathrm{ml}$ rabbit anti$\mathrm{a} 3(\mathrm{IV}) \mathrm{NC} 1 \mathrm{pAb}(50 \mu \mathrm{l} /$ well) for $1 \mathrm{~h}$ at RT. After washing with PBS-T, the wells were further incubated with 1:20,000 HRP-conjugated goat anti-rabbit IgG antibody ( $50 \mu \mathrm{l} /$ well) for $1 \mathrm{~h}$ at RT. After washing with PBST, 3,3c,5,5ל-tetramethylbenzidine (TMB) was added into the wells. After 30 min incubation at RT, $1 \mathrm{M}$ $\mathrm{H}_{2} \mathrm{SO}_{4}$ was added to stop the reaction. Absorbance at $450 \mathrm{~nm}$ was measured using Multiskan FC (Thermo Fisher Scientific). The optical density of the PC was set as 100 , and the relative values of samples (\% reactivity) were calculated.

\section{Detection of a3(IV)NC1 and macrophages in FFPE kidney sections of patients with renal disease by IHC}

FFPE kidney sections of patients with renal disease were autoclaved for $20 \mathrm{~min}$ at $121^{\circ} \mathrm{C}$ in Tris-EDTA $\left(\mathrm{pH} \mathrm{9.0)}\right.$ to retrieve the antigens. Thereafter, the sections were exposed to $3 \% \mathrm{H}_{2} \mathrm{O}_{2}$-methanol to inactivate endogenous peroxidase in the tissues followed by blocking the non-specific binding of antibodies and then allowed to react with rat anti-a3(IV)NC1 mAb (1:1000) for $1 \mathrm{~h}$ at RT. As a control, isotype rat IgG2a 
(MBL, Nagoya, Japan) was used. After rinsing with PBS, the sections were next made to react with the HRP-conjugated goat anti-rat IgG2a antibody (1:1000) for $1 \mathrm{~h}$ at RT. After rinsing with PBS, the HRP activity on tissue sections was detected by DAB substrates followed by counterstaining with Mayer's hematoxylin dye.

In other experiments, the sections stained for a3(IV)NC1 were further allowed to react with rabbit antiCD11c pAb (1:500) for $1 \mathrm{~h}$ at RT before counterstaining. After rinsing with PBS, the sections were next made to react with alkaline phosphatase (AP)-polymer reagents (Dako) for $1 \mathrm{~h}$ at RT. After rinsing with PBS, the AP activity on tissue sections was detected by fuchsin chromogen followed by counterstaining with Mayer's hematoxylin dye.

Alternatively, FFPE kidney sections of patients with renal disease were autoclaved for $20 \mathrm{~min}$ at $121^{\circ} \mathrm{C}$ in citrate buffer $(\mathrm{pH} 6.0)$ to retrieve the antigens. Thereafter, the sections were exposed to $3 \% \mathrm{H}_{2} \mathrm{O}_{2}$-methanol to inactivate endogenous peroxidase in the tissues followed by blocking the non-specific binding of antibodies and then allowed to react with mouse anti-CD68 mAb (1:50) for $1 \mathrm{~h}$ at RT. After rinsing with PBS, the sections were next made to react with the HRP-conjugated goat anti-mouse IgG antibody (1:100) for $1 \mathrm{~h}$ at RT. After rinsing with PBS, the HRP activity on tissue sections was detected by DAB substrates followed by counterstaining with Mayer's hematoxylin dye.

\section{Detection of a3(IV)NC1 and macrophages in FFPE kidney sections of patients with renal disease by immunofluorescence staining}

FFPE kidney sections of patients were autoclaved for $20 \mathrm{~min}$ at $121^{\circ} \mathrm{C}$ in Tris-EDTA (pH 9) to retrieve the antigens. Thereafter, the sections were subjected to blocking of the non-specific antibody binding and then allowed to react with rabbit anti-CD11c pAb (1:500) overnight at $4^{\circ} \mathrm{C}$. After rinsing with PBS, the sections were subsequently made to react with Alexa Fluor 594-conjugated goat anti-rabbit IgG antibody (1:1000) for $1 \mathrm{~h}$ at RT. The sections were next allowed to react with mouse anti-CD68 mAb (1:50) for $1 \mathrm{~h}$ at RT. After rinsing with PBS, the sections were subsequently made to react with Alexa Fluor 488conjugated goat anti-mouse IgG antibody (1:500) for $1 \mathrm{~h}$ at RT followed by mounting using a solution containing 4ל,6-diamidino-2-phenylindole (DAPI; Vector Laboratories, Burlingame, CA, USA).

In other experiments, after blocking of the non-specific antibody binding, FFPE kidney sections were reacted to mouse anti-tumstatin, a3(IV)NC1 mAb (1:500), and rabbit anti-CD11c pAb (1:500) overnight at $4^{\circ} \mathrm{C}$. After rinsing with PBS, the sections were next made to react with Alexa Fluor 488-conjugated goat anti-mouse IgG (1:500) and Alexa Fluor 594-conjugated goat anti-rabbit IgG (1:1000) antibodies for $1 \mathrm{~h}$ at RT followed by mounting using the solution containing DAPI.

\section{Making of AAV model rats}

According to Little's protocol [13], WKY rats (4 weeks old, male) were immunized with human native MPO $(1600 \mu \mathrm{g} / \mathrm{kg})$ on day 0 and given an intraperitoneal injection of pertussis toxin ( $800 \mathrm{ng})$ on days 0 and 2 (Group 1, original protocol, $n=11$ ). Because the administration of PMA has been shown to exacerbate 
renal disorder in other AAV model rats [14], PMA was employed to modify the original protocol. Materially, WKY rats immunized with human MPO were further given an intraperitoneal injection of PMA $(1 \mu \mathrm{g})$ on days $7,14,21,28$, and 35 (Group 2, modified protocol, $n=12$ ). For controls, rats were similarly immunized with or without recombinant human MPO light chain, which has been shown to induce non-pathogenic ANCA [15].

\section{Urinalysis and serological and histopathological examinations of AAV model rats}

All rats were euthanized on day 42 for blood and tissue sampling. Chronological blood sampling by tail cut was carried out on days 0,14 , and 28 . Urine samples were collected using a metabolic cage on day 40. Hematuria was assessed at urine sampling immediately by a dipstick (Siemens Healthineers, Erlangen, Germany). The serum was separated by centrifugation. ANCA was detected quantitatively using flow cytometry (FCM). In brief, human peripheral blood neutrophils were fixed with $4 \%$ paraformaldehyde, and then the plasma membrane of neutrophils was penetrated using Permeabilization Wash Buffer (BioLegend, San Diego, CA, USA). Cells $\left(1 \times 10^{6} / \mathrm{ml}\right)$ were allowed to react with 1:500 diluted rat sera for 30 min at RT. After washing with the buffer, cells were next made to react with $4 \mu \mathrm{g} / \mathrm{ml} \mathrm{FITC-conjugated}$ rabbit anti-rat IgG antibody for $30 \mathrm{~min}$ at RT. After washing with the buffer, cells were subjected to FCM using an Attune Acoustic Focusing Cytometer (Applied Biosystems, Foster City, CA, USA). Serum Creactive protein (CRP) levels at the time of death were determined by a commercial laboratory service (Daiichi Kishimoto Clinical Laboratory, Sapporo, Japan).

FFPE tissues of the systemic organs were cut into $4 \mu \mathrm{m}$ sections, deparaffinized with xylene, and then subjected to hematoxylin and eosin (HE) staining. Erythrocyte casts were counted in the maximum longitudinal section of the kidney specimen.

\section{Detection of anti-GBM antibody in rat sera}

Wells of 96-well plates were coated with $2.5 \mu \mathrm{g} / \mathrm{ml}$ recombinant human a3(IV)NC1 that exhibits a high homology with the rat counterpart ( $50 \mu \mathrm{l} /$ well) overnight at $4^{\circ} \mathrm{C}$ and then washed four times with PBS-T and soaked in PBS-T containing $1 \%$ skim milk (150 $\mu \mathrm{l} /$ well) for $1 \mathrm{~h}$ at RT to block the non-specific antibody binding. After the removal of the blocking solution, 1:10 diluted rat sera ( $50 \mu \mathrm{l} /$ well) were added into the wells and then incubated for $1 \mathrm{~h}$ at RT. After washing with PBS-T, the wells were next incubated with 1:10,000 diluted HRP-conjugated goat anti-rat IgG antibody (50 $\mu \mathrm{l} /$ well) for $1 \mathrm{~h}$ at RT. After washing with PBS-T, TMB was added into the wells. After 30 min incubation at RT, $1 \mathrm{M} \mathrm{H}_{2} \mathrm{SO}_{4}$ was added to stop the reaction. Absorbance at $450 \mathrm{~nm}$ was measured using Multiskan FC. To determine IgG subclass of anti-GBM antibody, 1:20,000 diluted HRP-conjugated anti-rat IgG1, IgG2a, and IgG2b antibodies were used as secondary antibodies.

\section{IHC for rat tissues}

FFPE kidney sections of normal rats were cut into $4 \mu \mathrm{m}$ sections, mounted on silane-coated glass slides, deparaffinized with xylene, and then heated using a water bath for $20 \mathrm{~min}$ at $95^{\circ} \mathrm{C}$ in citrate buffer $(\mathrm{pH}$ 
6.0) or autoclaved for $6 \mathrm{~min}$ at $105^{\circ} \mathrm{C}$ in $0.2 \mathrm{M} \mathrm{HCl}(\mathrm{pH} \mathrm{0.9)}$. These sections were subjected to $\mathrm{IHC}$ for a3(IV)NC1. FFPE kidney sections of AAV rats were deparaffinized with xylene and then heated using a water bath for 20 min at $95^{\circ} \mathrm{C}$ in citrate buffer (pH 6.0) to retrieve a3(IV)NC1 and CD11c or Tris-EDTA (pH 9.0) to retrieve $\mathrm{CD} 68$. Thereafter, the sections were exposed to $3 \% \mathrm{H}_{2} \mathrm{O}_{2}$-methanol to inactivate endogenous peroxidase in the tissues followed by blocking the non-specific binding of antibodies and then allowed to react with mouse anti-tumstatin, a3(IV)NC1 mAb $(1 \mu \mathrm{g} / \mathrm{ml})$, rabbit anti-CD11c pAb (1:100), or mouse anti-CD $68 \mathrm{mAb}(1: 50)$ for $1 \mathrm{~h}$ at RT. These anti-human antibodies have been confirmed to react with each rat counterpart. After rinsing with PBS, the sections were next made to react with the HRP-conjugated goat anti-mouse IgG antibody (1:100) or the HRP-conjugated goat anti-rabbit IgG antibody (1:500) for $1 \mathrm{~h}$ at RT. After rinsing with PBS, the HRP activity on tissue sections was detected by DAB substrates followed by counterstaining with Mayer's hematoxylin dye.

\section{Statistics}

Student's $t$-test and Mann-Whitney U-test were applied for comparison between parametric and nonparametric groups, respectively. $p<0.05$ was regarded as statistically significant.

\section{Results}

\section{Retrieval of a3(IV)NC1 from FFPE normal kidney sections by protease digestion in IHC}

First, the retrieval of a3(IV)NC1 from FFPE normal kidney sections in IHC was examined using anti$a 3(I V) N C 1$ mAb. This mAb recognizes a cryptic IPSTVKA amino acid sequence in the a3(IV)NC1 region. Although the anti-a3(IV)NC1 mAb did not bind to the epitope without antigen retrieval (Fig. 1A), a clear reaction with GBM and the basement membrane of Bowman's capsule that connects to the glomerulus was observed when the sections were heated under acidic condition ( $\mathrm{pH} 0.9$; Fig. 1B). This linear staining pattern was consistent with the characteristic distribution of GBM antigens that was recognized by the sera of patients with anti-GBM disease [16]. In contrast, no reaction appeared when the sections were heated under neutral condition ( $\mathrm{pH}$ 6.0; Fig. 1C) or alkaline condition (pH 9.0; Fig. 1D). Interestingly, the anti-a3(IV)NC1 mAb bound to GBM in FFPE normal kidney sections when the sections were exposed to proteases derived from $S$. griseus after heating under neutral condition (Fig. 1E). Although the nuclear staining by hematoxylin in Fig. 1B was weaker than that in Fig. 1E, Col (IV) was stained equivalently between the two. These findings corresponded to previous reports $[10,12]$ and suggested that proteases can digest $\mathrm{Col}(\mathrm{IV})$, resulting in the reveal of the cryptic a3(IV)NC1.

\section{Reveal of a3(IV)NC1 by protease digestion of Col (IV)}

Protease treatment that aims to digest formalin-mediated protein bridges is one of the common methods for antigen retrieval in IHC. To determine that the proteases derived from S. griseus digested not only formalin-mediated protein bridges but also Col (IV) in FFPE normal kidney sections and consequently revealed a3(IV)NC1, whether a3(IV)NC1 could be revealed by the protease digestion of Col (IV) was assessed by ELISA. Materially, Col (IV) was digested by diverse concentrations of proteases, and then the 
samples were subjected to ELISA for a3(IV)NC1 detection. Results demonstrated that a3(IV)NC1 was detected when Col (IV) was digested by an appropriate concentration $(125 \mu \mathrm{g} / \mathrm{ml})$ of the proteases derived from S. griseus (Fig. 1F). Because the proteases derived from S. griseus possess at least three caseinolytic activities and one aminopeptidase activity, it seems likely that a3(IV)NC1 itself was digested at concentrations higher than $125 \mu \mathrm{g} / \mathrm{ml}$. Next, a similar examination was carried out using a neutrophilspecific protease, NE, instead of the $S$. griseus-derived general proteases. The reveal of a3(IV)NC1 was also observed when Col (IV) was digested by NE in a dose-dependent manner (at a concentration of ${ }^{3} 500$ $\mu \mathrm{U} / \mathrm{ml}$; Fig. 1G). These findings suggested that proteases, including NE, can digest Col (IV) and result in the reveal of a3(IV)NC1.

\section{Reactivity of anti-a3(IV)NC1 antibody with GBM in FFPE kidney sections of patients}

It was hypothesized that, in AAV, proteases, including NE, derived from neutrophils activated by ANCA could digest Col (IV) of GBM. To elucidate this hypothesis, whether the anti-a3(IV)NC1 mAb could react with GBM in FFPE kidney sections of patients with MPA was assessed by IHC. As a histopathological feature of MPA, glomeruli showed diverse remarks from unaffected to global sclerosis. As shown in Fig. $2 A$, any specific binding of the $\mathrm{mAb}$ to unaffected glomeruli did not appear when the sections were heated under alkaline condition and not treated by proteases. In contrast, weak binding of the mAb to GBM was observed in crescentic glomeruli of MPA without any particular antigen retrieval (Fig. 2B). Weak binding of the mAb to GBM was also observed in crescentic glomeruli of IgA nephritis (IgAN; Fig. 2C). Moreover, an obvious binding of the anti-a3(IV)NC1 mAb was observed in the sclerotic glomeruli of MPA (Fig. 2D), IgAN (Fig. 2E), and hypertensive arteriosclerosis (Fig. 2F). Although the typical linear staining of GBM was blurred on the destructed glomeruli, the specificity of the antibody binding was guaranteed because there was no background staining when isotype control rat IgG2a was used as the primary antibody (Figs. 2B and 2D, inset). Although these findings are consistent with the authors' hypothesis in part, the reveal of a3(IV)NC1 in affected glomeruli appears not only in MPA but also in other renal disorders.

\section{Distribution of $\mathrm{CD} 11 \mathrm{c}^{+}$macrophages}

Although the reveal of a3(IV)NC1 in destructed glomeruli was not specific to AAV, the secondary production of anti-GBM antibody rarely occurs in diseases other than AAV. Therefore, the focus was next set on antigen-presenting cells (APCs). It has been shown that tissue-infiltrating CD $11 \mathrm{c}^{+}$macrophages play roles in antigen presentation [17]. IF staining demonstrated the presence of cell-surface CD11 $\mathrm{c}^{+}$and cytoplasmic $\mathrm{CD} 68^{+}$macrophages in glomeruli, Bowman's capsules, and renal tubules in MPA (Fig. 3A). In contrast, infiltration of $\mathrm{CD}_{11 \mathrm{c}^{+}}$macrophages was not apparent in kidney tissues of patients with IgAN and hypertensive arteriosclerosis (Figs. 3B-3D), although there were a certain amount of $C D 68^{+}$ macrophages, which indicates active state of the diseases (Figs. 3B, 3C, and 3E).

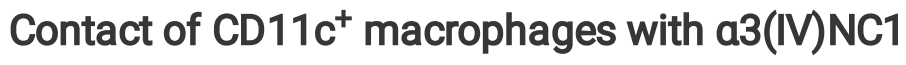


The association of $\mathrm{CD}_{11} \mathrm{c}^{+}$macrophages with a3(IV)NC1 was examined. IF staining demonstrated that

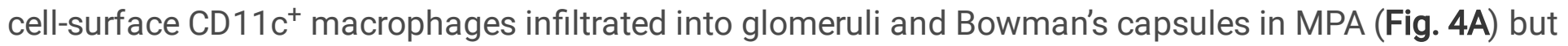
not in IgAN (Fig. 4B) or hypertensive arteriosclerosis (Fig. 4C). Furthermore, some CD11 $\mathrm{c}^{+}$macrophages, which infiltrated into the lesions of MPA, were present, near a3(IV)NC1, and took it into their cytoplasm (Fig. 4D).

\section{Establishment of AAV rat model with exacerbation of renal tissue damage}

Although WKY rats immunized with human native MPO are useful models of AAV [13], the renal manifestation is mild compared to typical MPA in humans. To make the renal disorder worse in this model, the original protocol was modified using PMA based on the authors' previous study, demonstrating that PMA administration exacerbated renal disorders in the drug-induced AAV rat model [14]. WKY rats $(n=23)$ were immunized with human MPO according to the original protocol [13] and then divided into two groups, namely, Group 1 without PMA administration (original protocol, $n=11$ ) and Group 2 with PMA administration (modified protocol, $n=12$ ). Group 2 rats were given an intraperitoneal injection of PMA $(1 \mu \mathrm{g})$ on days $7,14,21,28$, and 35.

All rats in Groups 1 and 2 produced ANCA, and the titer was comparable between the two (Figs. 5A and 5B). Severe hematuria was detected in both groups of rats (Fig. 5C, left), whereas CRP levels were significantly higher in Group 2 than in Group 1 (Fig. 5C, middle). Correspondingly, the degree of renal tissue destruction represented by the number of erythrocyte casts in the kidney sections tended to be larger in Group 2 compared to Group 1 (Figs. 5C, right, and 5D). Infiltration of neutrophils into the upstream renal tubules of the erythrocyte casts was observed (Fig. 5D, inset). Although both groups of rats did not develop overt crescentic glomerulonephritis, the collective findings indicated that renal tubular disorder was exacerbated in the AAV rat model by PMA.

\section{Production of anti-GBM antibody in exacerbated AAV model rats}

To determine the production of anti-GBM antibody in AAV models, serum samples on days 0 and 42 were subjected to ELISA (Fig. 6A). Anti-GBM antibody was not detected in all day 0 sera $(n=23)$. Whereas neither rats in Group 1 nor rats given intraperitoneal PMA injection with mock-immunization produced anti-GBM antibody, two rats in Group 2 (\#6 and \#9) produced anti-GBM antibody. Interestingly, chronological analysis of autoantibody production revealed that anti-GBM antibody production followed ANCA production in these rats (Fig. 6B). Also, the subclass of anti-GBM antibody produced in Group 2 AAV rats was determined as IgG2a, one of the pathogenic subclasses [18] (Fig. 6C).

Interstitial tubular rather than glomerular damage was exacerbated in AAV rats by PMA administration. Contrary to humans, Col (IV), including a3(IV)NC1, was distributed in the basement membrane of tubules as well as in glomeruli in rats (Fig. 6D). This finding corresponded to the literature reporting that the a3subunit of Col (IV) is distributed in the basement membrane of renal tubules in rats [19]. IHC demonstrated the reveal of a3(IV)NC1 in the affected renal interstitial tissues of Group 2 rats with anti- 
GBM antibody production and infiltration of $\mathrm{CD}_{11} \mathrm{c}^{+}$macrophages around the sites but not in Groups 1 and 2 rats without anti-GBM antibody production (Fig. 6E).

\section{Discussion}

Anti-GBM disease is an organ-specific autoimmune disorder characterized by anti-GBM antibodymediated diffuse necrotizing crescentic glomerulonephritis and pulmonary hemorrhage [3]. A linear IgG deposition along the autologous GBM-usually detected by IF staining using frozen sections of a biopsied kidney-is a diagnostic hallmark of this disease. However, anti-GBM antibody in patients' serum cannot bind to GBM in FFPE normal kidney sections because a3(IV)NC1, which covers the major epitopes of anti-GBM antibody, is structurally cryptic $[5,6,10]$. Although a contribution of the anti-peroxidasin antibody to the misfolding of the sulphilimine linkage of 3-4-5 a-subunit trimers of NC1 domain, which conceals a3(IV)NC1, has been suggested [20], the mechanism of the reveal of the cryptic epitopes in patients with anti-GBM disease remains elusive.

Based on the clinical observation of the close association between anti-GBM disease and AAV [7, 8], it was hypothesized that proteases released from neutrophils activated by ANCA could digest Col (IV) and consequently reveal cryptic epitopes. In this study, it was successfully demonstrated that (1) a3(IV)NC1 was revealed by the digestion of Col (IV) by proteases, including NE; (2) although the reveal of a3(IV)NC1 was observed in affected glomeruli regardless of causative diseases, that accompanied by CD11 $\mathrm{c}^{+}$ macrophage infiltration was a characteristic of MPA (AAV); and (3) anti-GBM antibody was produced subsequent to ANCA in some AAV model rats.

The results of ELISA have demonstrated that $500 \mu \mathrm{U} / \mathrm{ml}$ or higher concentrations of NE are required to reveal a3(IV)NC1 from Col (IV). Concerning this issue, we have assumed that NE released from ANCAactivated neutrophils digests GBM in the glomerular microenvironment.

The reveal of a3(IV)NC1 was observed in affected glomeruli regardless of causative diseases, including IgAN and hypertensive arteriosclerosis. It has been shown that matrix metalloproteinase-9 is involved in the formation of mesangial lesions in IgAN [21] and that mast cell-derived tryptase and chymase are implicated in the development of hypertensive nephropathy [22]. Similarly to the neutrophil-derived proteases in AAV, these proteases may contribute to reveal a3(IV)NC1 due to the destruction of glomeruli while sclerosis occurs. However, this study has demonstrated that the reveal of a3(IV)NC1 in affected glomeruli accompanied by $\mathrm{CD}_{11 \mathrm{c}^{+}}$macrophage infiltration was a characteristic of MPA (AAV). In addition, it was noted that some $\mathrm{CD} 11 \mathrm{c}^{+}$macrophages, which infiltrated into the lesions of MPA, have incorporated a3(IV)NC1 in the cytoplasm. Wlodarczyk et al. have demonstrated that tissue-infiltrating $\mathrm{CD}_{11 \mathrm{c}^{+}}$macrophages can act as APCs [17]. Although the pathway that leads to the anti-GBM antibody production has not been uncovered, it is assumed that the a3(IV)NC1-incorporated CD $11 \mathrm{c}^{+}$macrophages flow into the regional lymph nodes, present the GBM epitopes on class II major histocompatibility complex (MHC), and then helper T-cells control the subsequent immune reaction. Discovery of the mechanism is a critical future subject. 
Interestingly, anti-GBM antibody was produced in some AAV model rats made by the modified protocol with additional injections of PMA. PMA administration exacerbated the destruction of renal tubules in AAV model rats. When the recombinant human MPO light chain, instead of native MPO, was used as an immunogen, ANCA was not detected by FCM [15], and renal tissue destruction was minimal regardless of PMA administration (unpublished results). Serum CRP levels were not elevated significantly in the rats immunized with the non-pathogenic recombinant human MPO light chain followed by PMA administration. These findings suggested that PMA itself cannot induce renal tissue destruction if it is not accompanied by ANCA, which can bind neutrophil MPO, and that the elevated CRP levels in Group 2 rats can be reflected by renal interstitial inflammation. PMA could induce the priming of neutrophils [23], enhance the ANCA-mediated activation of these cells, and contribute to the exacerbation of renal tissue destruction. As Col (IV), including a3(IV)NC1, is distributed in the basement membrane of renal tubules in rats, the reveal of a3(IV)NC1 in the affected interstitial space of renal tubules is reasonable.

Based on the collective findings, it was suggested that, in AAV, proteases released from neutrophils activated by ANCA digest Col (IV) and result in the reveal of a3(IV)NC1, AAV-specific CD11 $\mathrm{c}^{+}$ macrophages catch and present the epitopes in the region, and then the host's immune system produces anti-GBM antibody (Fig. 7). This corresponded to the concept of intermolecular epitope spreading.

Epitope spreading was first described in autoimmune models of multiple sclerosis (MS) [24]. This phenomenon involves the following steps: tissue destruction by a primary immune response against a certain epitope, phagocytosis of tissue debris by APCs, and induction of secondary immune response against neo-epitopes [25]. Epitope spreading occurs both in a single antigen and among diverse antigens; the former is called intramolecular epitope spreading and the latter is called intermolecular epitope spreading. Intramolecular epitope spreading is associated with the step-by-step progression of the disease, such as MS. In contrast, intermolecular epitope spreading is related to disease conversion and complication. For example, the implication of intermolecular epitope spreading has been discussed in a conversion from pemphigus vulgaris to pemphigus foliaceus [26] and in a complication of Hashimoto's thyroiditis with Graves' disease [27]. To the authors' knowledge, this is the first report suggesting that intermolecular epitope spreading is involved in the production of anti-GBM antibody in AAV.

All AAV patients do not produce an anti-GBM antibody regardless of the reveal of a3(IV)NC1 with a close

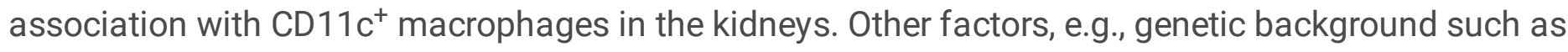
$\mathrm{MHC}$ haplotypes, might be involved in the mechanism of anti-GBM antibody production in AAV patients. In contrast, in AAV induced in inbred WKY rats, the severity of renal tissue destruction could be critically implicated in the production of anti-GBM antibody because the reveal of a3(IV)NC1 in affected renal tissues was detected only in rats with anti-GBM antibody production.

It has been shown that the complication of anti-GBM disease is linked to the poor prognosis of AAV [3]. In fact, $38 \%$ of anti-GBM antibody ${ }^{+}$AAV patients developed alveolar hemorrhage, while $23 \%$ of anti-GBM antibody ${ }^{-}$AAV patients did. It is necessary to determine the pathogenicity of anti-GBM antibody following ANCA in AAV rats in future studies. Because the subclass of IgG has been reported to be associated with 
the severity of anti-GBM disease [18], the subclass of anti-GBM antibody in AAV rats was examined by ELISA, and it was determined as IgG2a, one of the pathogenic subclasses. Also, one of the two AAV rats with anti-GBM antibody production developed severer pulmonary hemorrhage than other rats (unpublished results). Although further investigation is needed, this study paves the way for anti-GBM antibody production in AAV, which can be linked to the poor prognosis of AAV.

\section{Declarations}

\section{Ethical approval and consent to participate}

The use of human materials was permitted by the Ethics Committee of the Faculty of Health Sciences, Hokkaido University (permission nos. 17-85, 18-7, and 18-34). Experiments using rats were permitted by the Animal Experimentation Committee of Hokkaido University (permission no. 15-0034) and performed in accordance with the Guidelines for the Care and Use of Laboratory Animals of Hokkaido University.

\section{Consent for publication}

Not applicable.

\section{Availability of data and materials}

The data sheets used and/or analysed during the current study are available from the corresponding author on reasonable request.

\section{Competing interests}

None.

\section{Funding}

This work was supported by a grant from the Ministry of Health, Labour, and Welfare of Japan for Japan Research Committee of Intractable Vasculitis (JPVAP; A.I.).

\section{Author contributions}

Y.N., S.M., U.T., and A.I. designed research. Y.N., M.N., Y.T., R.H., K.S., and H.H. performed research. Y.N., M.N., S.M., D.N., S.T., U.T., and A.I. analyzed data. Y.N. and A.I. wrote the paper. All authors discussed the results and contributed to the preparation of the manuscript.

\section{Acknowledgements}

Not applicable.

\section{Authors' information}


Not applicable.

\section{References}

1. Jennette JC, Falk RJ, Bacon PA, Basu N, Cid MC, Ferrario F, et al. 2012 revised International Chapel Hill Consensus Conference Nomenclature of Vasculitides. Arthritis Rheum. 2013;65(1):1-11.

2. Nakazawa D, Masuda S, Tomaru U, Ishizu A. Pathogenesis and therapeutic interventions for ANCAassociated vasculitis. Nat Rev Rheumatol. 2019;15(2):91-101.

3. McAdoo SP, Tanna A, Hruskova Z, Holm L, Weiner M, Arulkumaran N, et al. Patients doubleseropositive for ANCA and anti-GBM antibodies have varied renal survival, frequency of relapse, and outcomes compared to single-seropositive patients. Kidney Int. 2017;92(3):693-702.

4. Khoshnoodi J, Pedchenko V, Hudson BG. Mammalian collagen IV. Microsc Res Tech. 2008;71(5):35770.

5. Pedchenko V, Bondar O, Fogo AB, Vanacore R, Voziyan P, Kitching AR, et al. Molecular architecture of the Goodpasture autoantigen in anti-GBM nephritis. N Engl J Med. 2010;363(4):343-54.

6. Vanacore R, Pedchenko V, Bhave G, Hudson BG. Sulphilimine cross-links in Goodpasture's disease. Clin Exp Immunol. 2011;164 Suppl 1:4-6.

7. Levy JB, Hammad T, Coulthart A, Dougan T, Pusey CD. Clinical features and outcome of patients with both ANCA and anti-GBM antibodies. Kidney Int. 2004;66(4):1535-40.

8. Olson SW, Arbogast CB, Baker TP, Owshalimpur D, Oliver DK, Abbott KC, et al. Asymptomatic autoantibodies associate with future anti-glomerular basement membrane disease. J Am Soc Nephrol. 2011;22(10):1946-52.

9. Hara M, Mase D, Inaba S, Higuchi A, Tanizawa T, Yamanaka N, et al. Immunohistochemical localization of glomerular basement membrane antigens in various renal diseases. Virchows Arch $\mathrm{A}$ Pathol Anat Histopathol. 1986;408(4):403-19.

10. Naito I, Ninomiya Y, Nomura S. Immunohistochemical diagnosis of Alport's syndrome in paraffinembedded renal sections: antigen retrieval with autoclave heating. Med Electron Microsc. 2003;36(1):1-7.

11. Emoto K, Yamashita S, Okada Y. Mechanisms of heat-induced antigen retrieval: does $\mathrm{pH}$ or ionic strength of the solution play a role for refolding antigens? J Histochem Cytochem. 2005;53(11):1311-21.

12. Guan $N, Y u L X, W u G H$, Xing Y, Ding J. Antigen retrieval with protease digestion applied in immunohistochemical diagnosis of Alport syndrome. Nephrol Dial Transplant. 2008;23(11):3509-13.

13. Little MA, Smyth L, Salama AD, Mukherjee S, Smith J, Haskard D, et al. Experimental autoimmune vasculitis: an animal model of anti-neutrophil cytoplasmic autoantibody-associated systemic vasculitis. Am J Pathol. 2009;174(4):1212-20.

14. Nakazawa D, Tomaru U, Suzuki A, Masuda S, Hasegawa R, Kobayashi T, et al. Abnormal conformation and impaired degradation of propylthiouracil-induced neutrophil extracellular traps: 
implications of disordered neutrophil extracellular traps in a rat model of myeloperoxidase antineutrophil cytoplasmic antibody-associated vasculitis. Arthritis Rheum. 2012;64(11):3779-87.

15. Nonokawa M, Suzuki K, Hayashi H, Nishibata Y, Masuda S, Nakazawa D, et al. Native myeloperoxidase is required to make the experimental vasculitis model. Arthritis Res Ther. 2019;21(1):296.

16. Nishibata Y, Masuda S, Nakazawa D, Tanaka S, Tomaru U, Nergui M, et al. Epitope recognized by anti-glomerular basement membrane (GBM) antibody in a patient with repeated relapse of anti-GBM disease. Exp Mol Pathol. 2019;107:165-70.

17. Wlodarczyk A, Lobner M, Cedile 0 , Owens T. Comparison of microglia and infiltrating CD $11 \mathrm{c}^{+}$cells as antigen presenting cells for $\mathrm{T}$ cell proliferation and cytokine response. J Neuroinflammation. 2014;11:57.

18. Zhao J, Yan Y, Cui Z, Yang R, Zhao MH. The immunoglobulin G subclass distribution of anti-GBM autoantibodies against rHa3(IV)NC1 is associated with disease severity. Hum Immunol. 2009;70(6):425-9.

19. Sugihara K, Sado Y, Ninomiya Y, Wada H. Experimental anti-GBM glomerulonephritis induced in rats by immunization with synthetic peptides based on six a chains of human type IV collagen. J Pathol. 1996;178(3):352-8.

20. McCall AS, Bhave G, Pedchenko V, Hess J, Free M, Little DJ, et al. Inhibitory Anti-Peroxidasin Antibodies in Pulmonary-Renal Syndromes. J Am Soc Nephrol. 2018;29(11):2619-25.

21. Danilewicz M, Wagrowska-Danilewicz M. Differential glomerular immunoexpression of matrix metalloproteinases MMP-2 and MMP-9 in idiopathic IgA nephropathy and Schoenlein-Henoch nephritis. Folia Histochem Cytobiol. 2010;48(1):63-7.

22. Welker $\mathrm{P}$, Kramer $\mathrm{S}$, Groneberg DA, Neumayer HH, Bachmann S, Amann K, et al. Increased mast cell number in human hypertensive nephropathy. Am J Physiol Renal Physiol. 2008;295(4):F1103-9.

23. Shida H, Nakazawa D, Tateyama Y, Miyoshi A, Kusunoki Y, Hattanda F, et al. The Presence of AntiLactoferrin Antibodies in a Subgroup of Eosinophilic Granulomatosis with Polyangiitis Patients and Their Possible Contribution to Enhancement of Neutrophil Extracellular Trap Formation. Front Immunol. 2016;7:636.

24. Lehmann PV, Forsthuber T, Miller A, Sercarz EE. Spreading of T-cell autoimmunity to cryptic determinants of an autoantigen. Nature. 1992;358(6382):155-7.

25. Vanderlugt CL, Miller SD. Epitope spreading in immune-mediated diseases: implications for immunotherapy. Nat Rev Immunol. 2002;2(2):85-95.

26. Kawana S, Hashimoto T, Nishikawa T, Nishiyama S. Changes in clinical features, histologic findings, and antigen profiles with development of pemphigus foliaceus from pemphigus vulgaris. Arch Dermatol. 1994;130(12):1534-8.

27. McLachlan SM, Nagayama Y, Pichurin PN, Mizutori Y, Chen CR, Misharin A, et al. The link between Graves' disease and Hashimoto's thyroiditis: a role for regulatory T cells. Endocrinology. 2007;148(12):5724-33. 


\section{Figures}
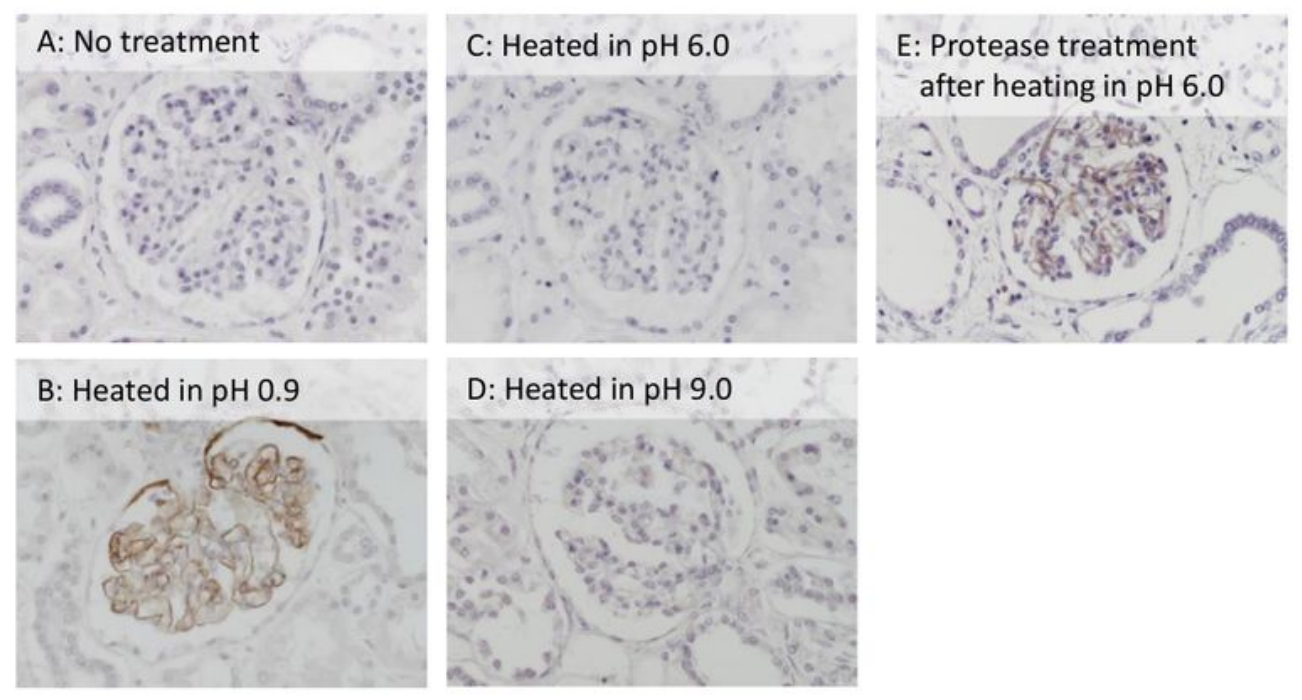

$\mathrm{F}$

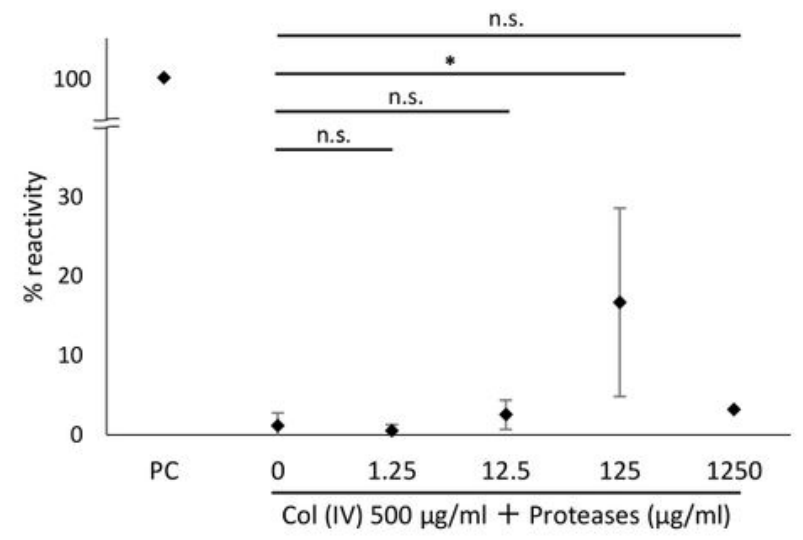

G

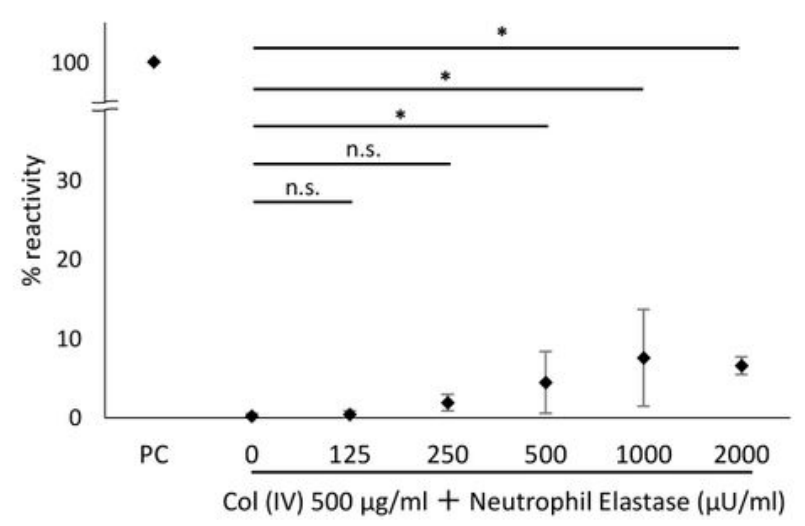

\section{Figure 1}

Reveal of a3(IV)NC1 by protease digestion FFPE normal kidney sections were subjected to IHC for a3(IV)NC1 (A-E): without heating (A), heated under acidic condition ( $\mathrm{pH} 0.9 ; \mathrm{B})$, heated under neutral condition ( $\mathrm{pH} 6.0 ; \mathrm{C})$, heated under alkaline condition ( $\mathrm{pH} 9.0 ; \mathrm{D})$, and protease treatment after heating 
under neutral condition (E). As general proteases, proteases derived from S. griseus were used. Representative microphotographs are shown. Col (IV) $(1 \mathrm{mg} / \mathrm{ml})$ was digested by proteases derived from S. griseus $(F)$ or $N E(G)$ for 30 min at $37^{\circ} \mathrm{C}$. Thereafter, the solution was heated for $15 \mathrm{~min}$ at $80^{\circ} \mathrm{C}$ to inactivate the proteases. Recombinant human a3(IV)NC1 $(1.25 \mu \mathrm{g} / \mathrm{ml})$ as a positive control (PC) and the samples were subjected to ELISA to detect a3(IV)NC1. The optical density of the PC was set as 100, and the relative values of samples (\% reactivity) were calculated. Experiments were repeated three times independently. Each experiment was conducted in duplicate. ${ }^{*} p<0.05 ;$ n.s., not significant.
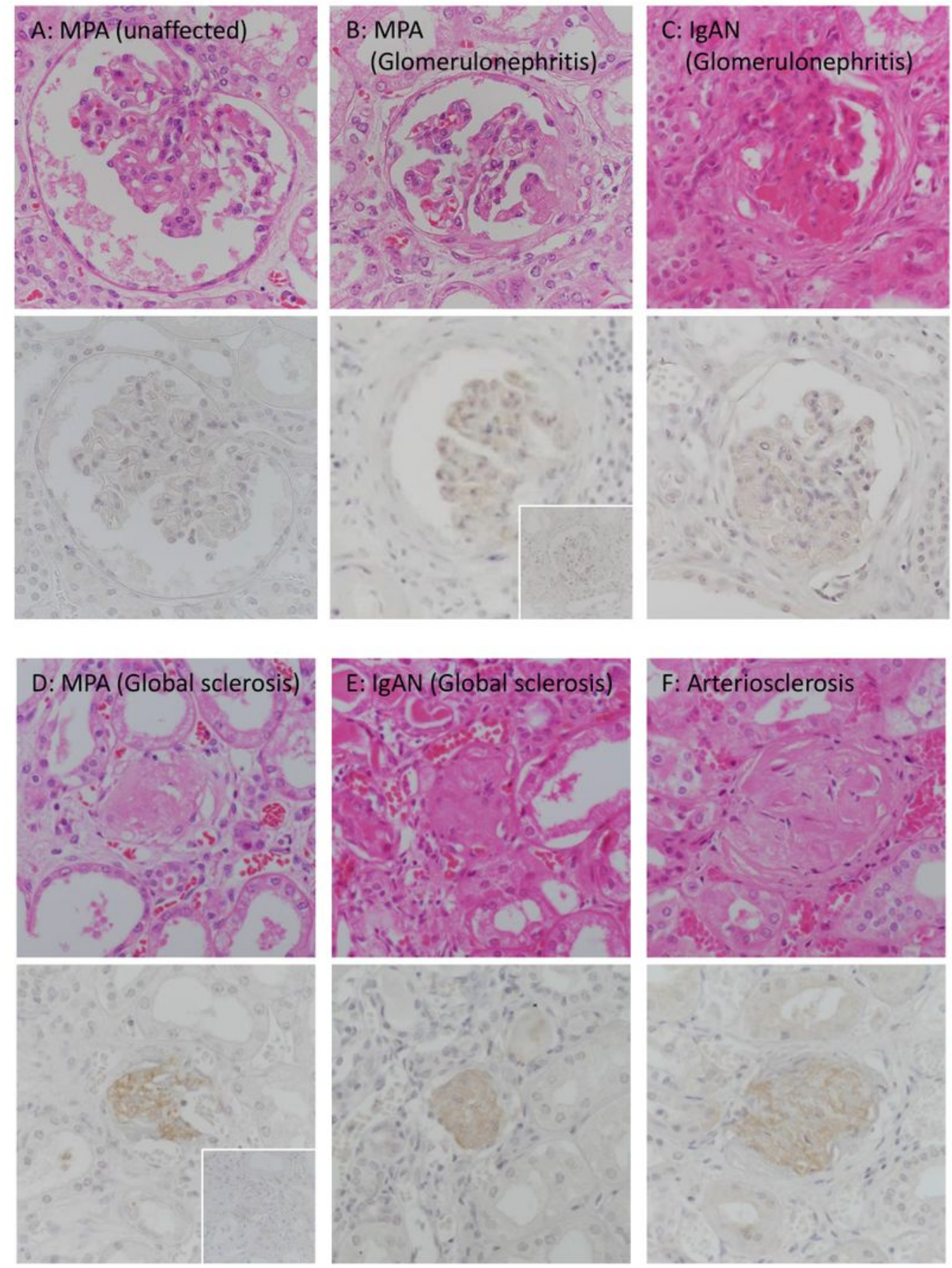
Figure 2

Reveal of a3(IV)NC1 in patients' kidneys HE staining and IHC for a3(IV)NC1 without any particular antigen retrieval using FFPE kidney sections of patients with MPA ( $A, B$, and D), IgAN ( $C$ and $E)$, and hypertensive arteriosclerosis $(F)$. Unaffected glomerulus $(A)$, crescentic glomerulus ( $B$ and $C$ ), and sclerotic glomerulus $(D-F)$. Representative microphotographs are shown. Insets in $B$ and $D$ represent background staining when rat lgG2a was used as isotype control.

A: MPA
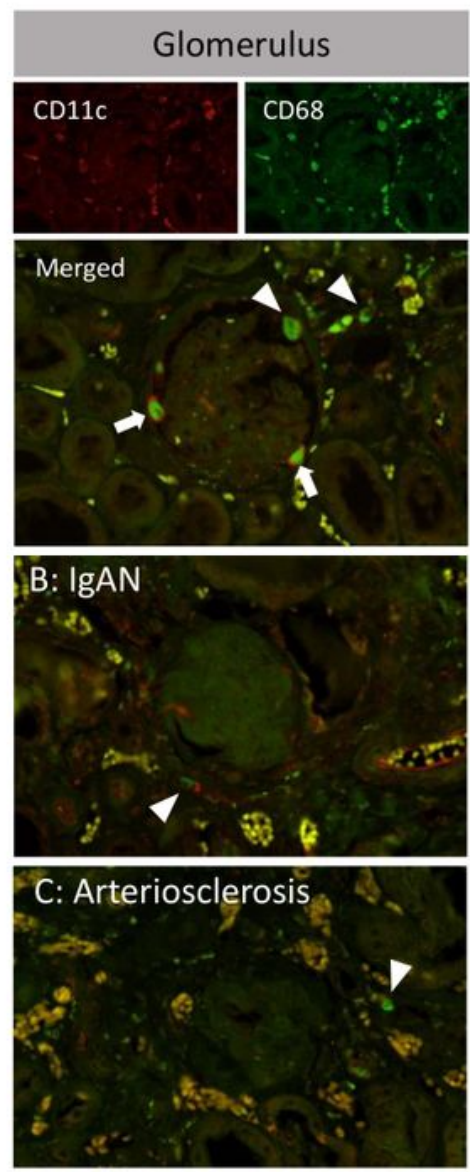
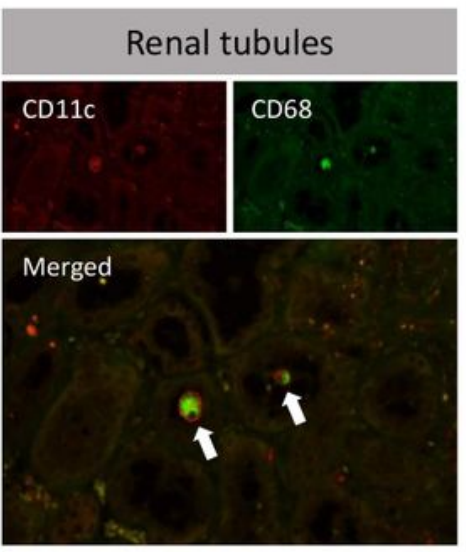

D

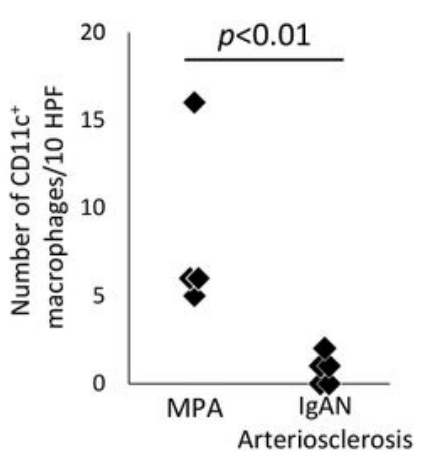

$E$

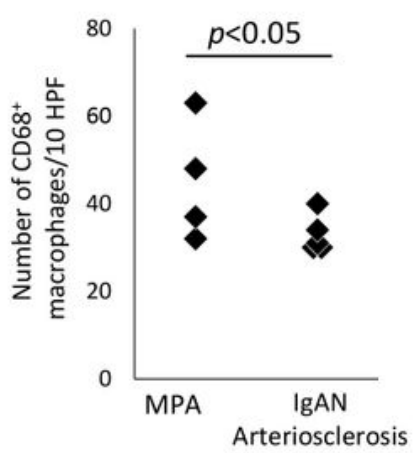




\section{Figure 3}

Infiltration of CD11C+ macrophages into kidneys IF staining for CD11c (red) and CD68 (green) using FFPE kidney sections of patients with MPA (A), IgAN (B), and hypertensive arteriosclerosis (C). Arrows indicate cell-surface CD11 + and cytoplasmic CD68+ macrophages. Arrowheads indicate CD68+ macrophages. Representative microphotographs are shown. The number of CD11c+ macrophages (D) and CD68+ macrophages (E) in 10 high-power fields focusing on glomeruli was counted and then compared between MPA and non-MPA (IgAN and hypertensive arteriosclerosis).

\section{A: MPA}
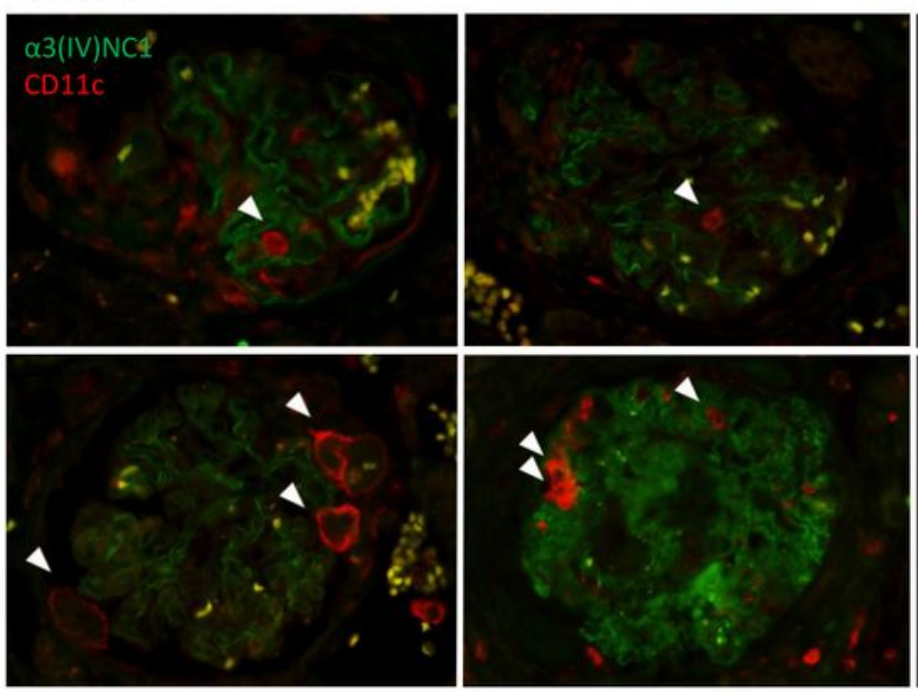

\section{B: IgAN}

C: Arteriosclerosis

\section{D: MPA}
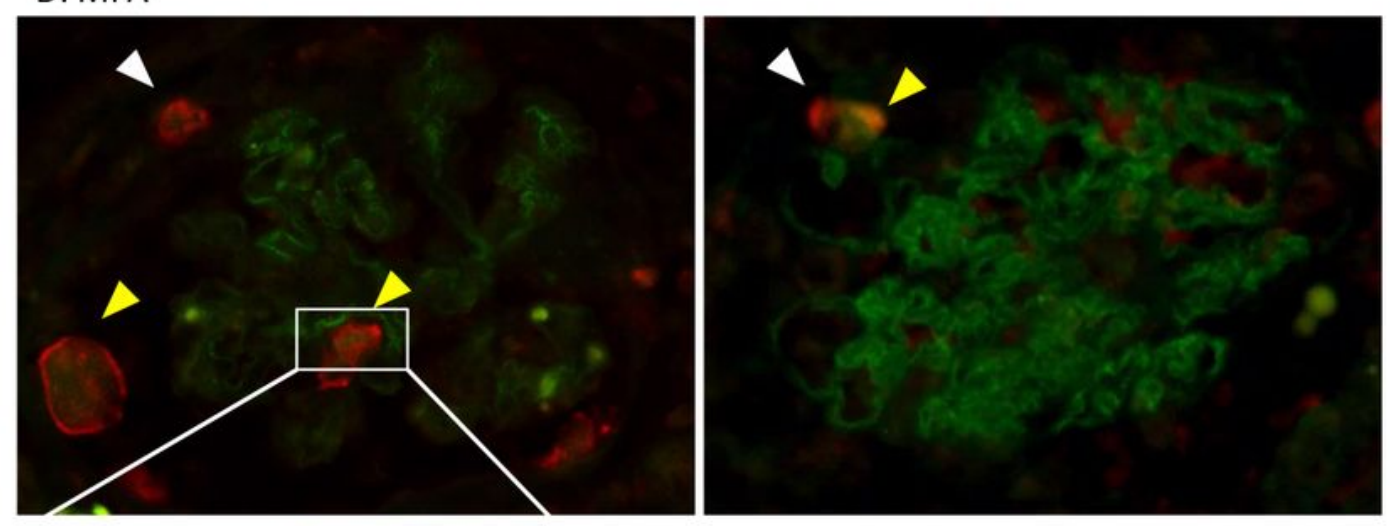

CD11c 
Figure 4

Contact of CD11c+ macrophages with a3(IV)NC1 IF staining for CD11c (red) and a3(IV)NC1 (green) using FFPE kidney sections of patients with MPA (A and D), IgAN (B), and hypertensive arteriosclerosis (C). White arrowheads indicate $\mathrm{CD} 11 \mathrm{c}+$ macrophages, and yellow arrowheads indicate those that take a3(IV)NC1 into their cytoplasm (D). Representative microphotographs are shown.

A

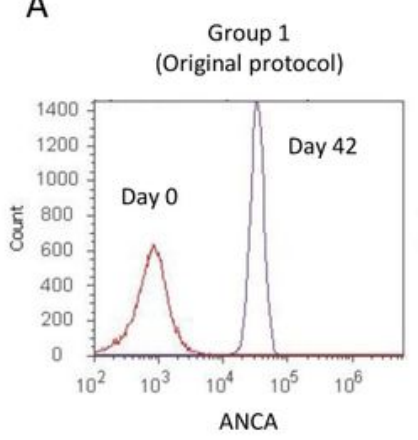

C

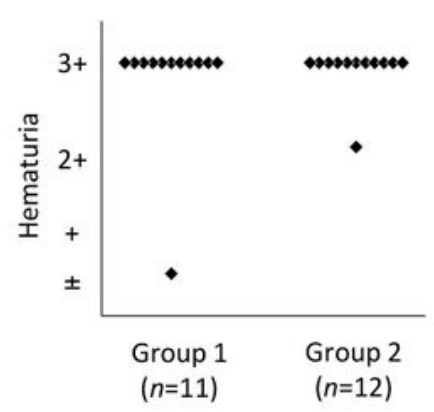

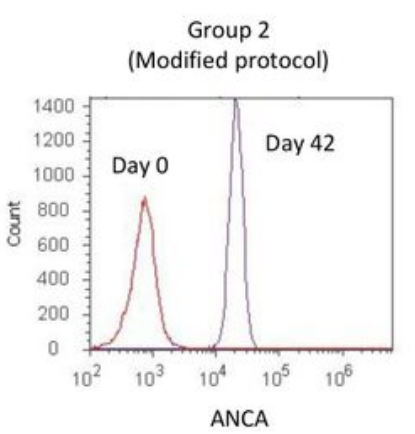

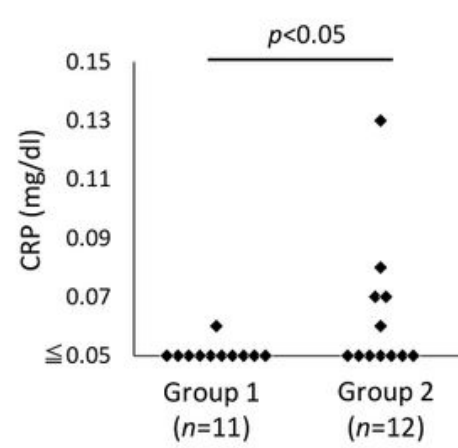

B

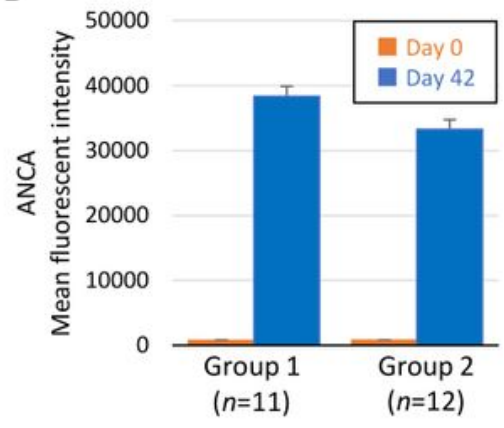

D
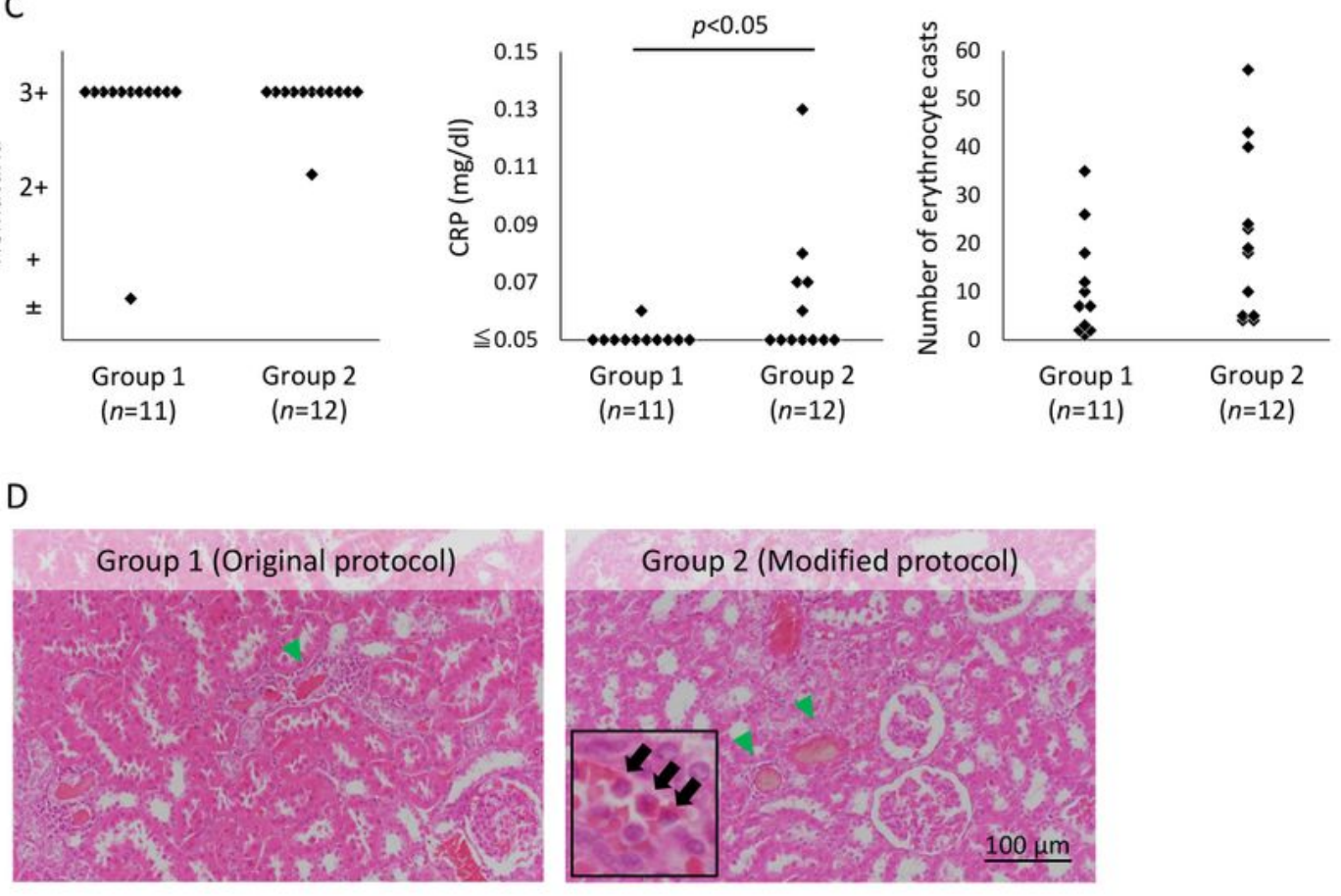

D

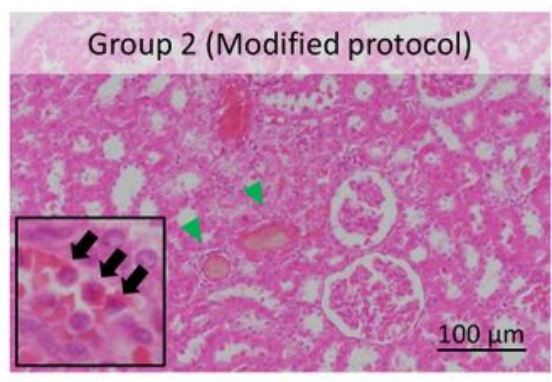


AAV induction in rats WKY rats $(\mathrm{n}=23)$ were immunized with human MPO and then divided into two groups, namely, Group 1 without PMA administration (original protocol, $\mathrm{n}=11$ ) and Group 2 with PMA administration (modified protocol, $n=12$ ). Group 2 rats were given an intraperitoneal injection of PMA ( 1 $\mu \mathrm{g}$ ) on days $7,14,21,28$, and 35 . ANCA detection by FCM (A and B). Comparison of hematuria, serum levels of CRP, and the number of erythrocyte casts in the maximum longitudinal section of the kidney specimen between Groups 1 and 2 (C). HE staining showing renal damage represented by erythrocyte casts (green arrowheads; D). Arrows in the inset indicate neutrophils that infiltrated into the upstream renal tubules of the erythrocyte casts. Representative microphotographs are shown. 


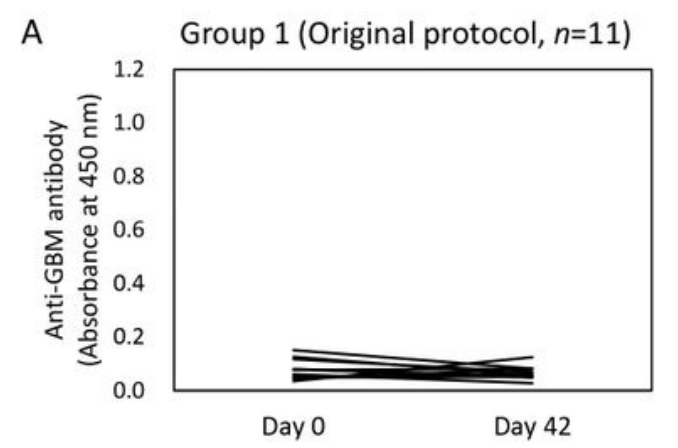

Group 2 (Modified protocol, $n=12$ )
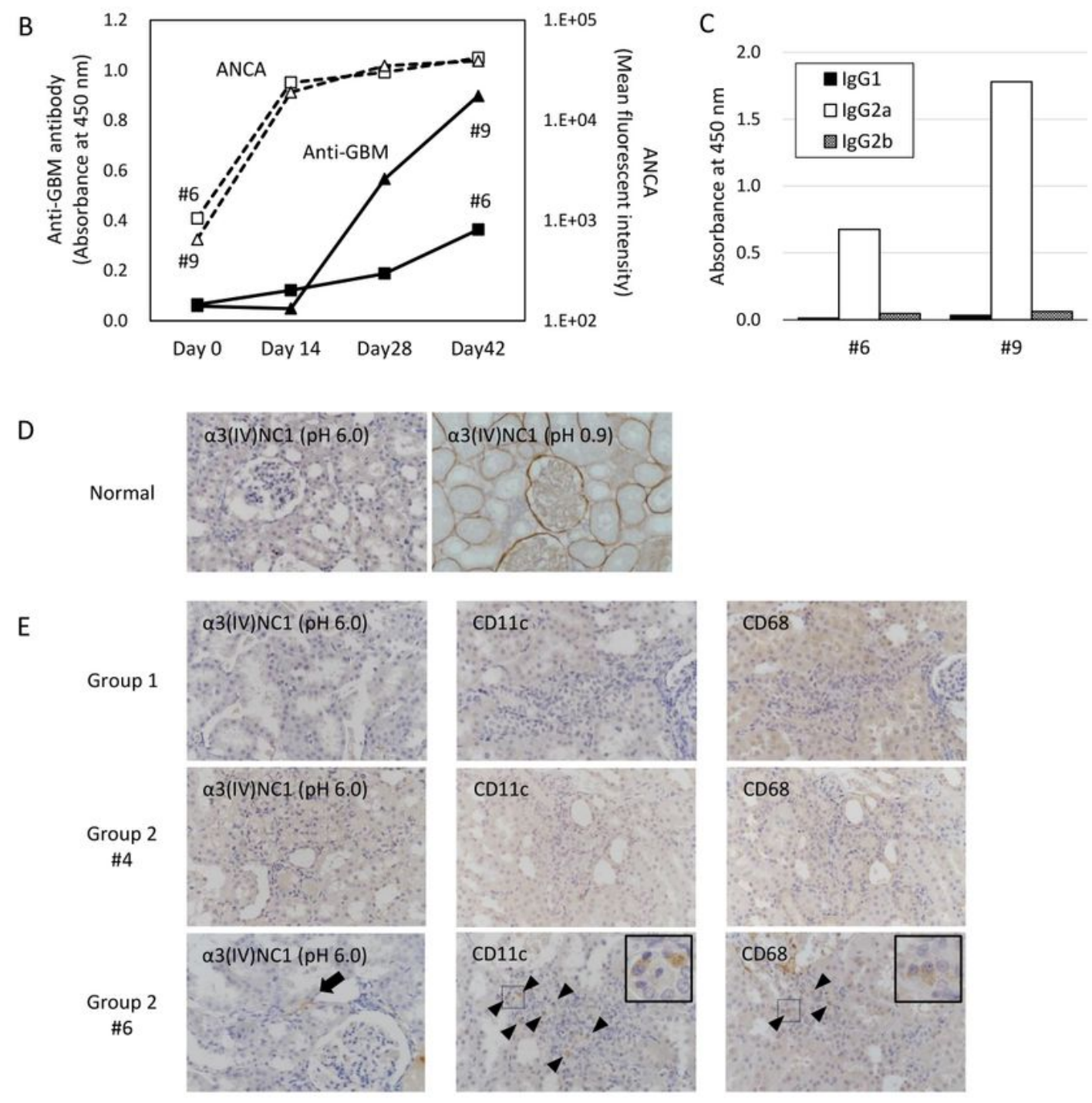

\section{Figure 6}

Production of anti-GBM antibody in AAV model rats Anti-GBM antibody in sera was measured by ELISA (A). Anti-GBM antibody was not detected in all day 0 sera. Whereas no rat in Group 1 produced anti-GBM antibody, two rats in Group 2 (\#6 and \#9) produced anti-GBM antibody. Chronological production of ANCA and anti-GBM antibody in \#6 and \#9 rats in Group 2 (B). ANCA titer was determined as the mean fluorescent intensity of FCM. The IgG subclass of anti-GBM antibody was determined by ELISA (C). 
Distribution of a3(IV)NC1 in normal rat kidney sections (D). FFPE kidney sections of normal rats were heated at pH 6.0 (left) or pH 0.9 (right) and then subjected to IHC for a3(IV)NC1. The reveal of a3(IV)NC1 (black arrow) and the infiltration of CD11c+ and CD68+ cells (black arrowheads) were observed in the kidneys of Group 2 rats with anti-GBM antibody production (\#6) but not in the kidneys of Groups 1 rats and Group 2 rats without anti-GBM antibody production (\#4) (E). Representative microphotographs are shown.

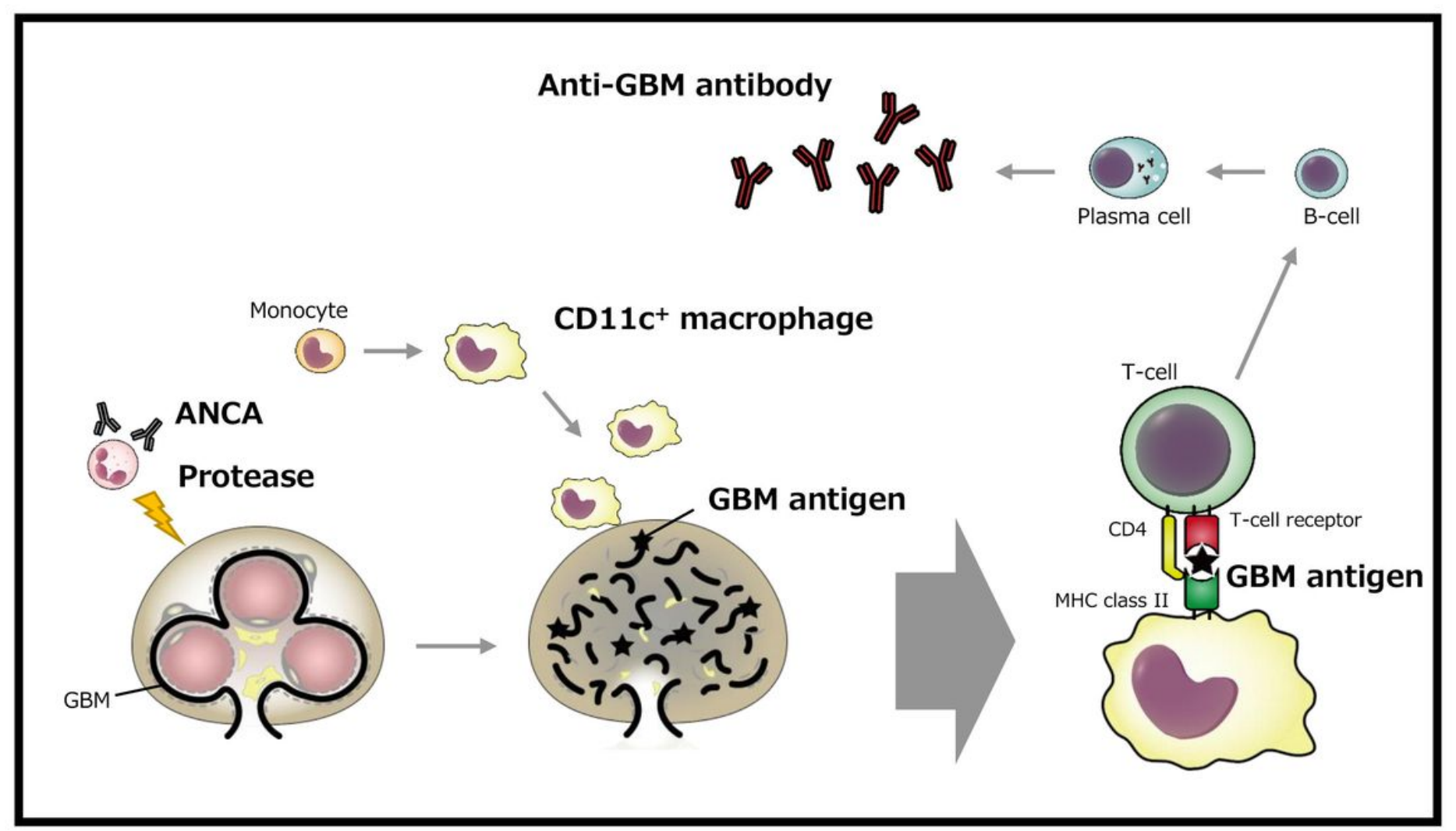

Figure 7

A schema of the production mechanism of anti-GBM antibody in AAV In AAV, proteases released from neutrophils activated by ANCA digest Col (IV) and result in the reveal of a3(IV)NC1, AAV-specific CD11c+ macrophages catch and present the GBM epitopes, and then the host's immune system produces antiGBM antibody. 